\title{
Effect of pegbovigrastim administration on the microbiome found in the vagina of cows postpartum
}

\author{
K. N. Galvão, ${ }^{1}$ C. H. Higgins, ${ }^{2}$ M. Zinicola, ${ }^{2}$ S. J. Jeon, ${ }^{1}$ H. Korzec, ${ }^{2}$ and R. C. Bicalho ${ }^{2 *}$ \\ ${ }^{1}$ Department of Large Animal Clinical Sciences and D. H. Barron Reproductive and Perinatal Biology Research Program, University of Florida, \\ Gainesville 32610 \\ ${ }^{2}$ Department of Population Medicine and Diagnostic Sciences, Cornell University, Ithaca, NY 14853-6401
}

\section{ABSTRACT}

The objective of this study was to evaluate the effect of pegbovigrastim (PEG) treatment of peripartum Holstein cows on the microbiome found in the vagina postpartum using sequencing of the $16 \mathrm{~S}$ rRNA gene. A subset of cows was randomly sampled from a larger study where cows had been randomly assigned to 1 of 2 treatments: pegbovigrastim (PEG) or untreated control (CTR). The PEG-treated cows received a subcutaneous injection containing $15 \mathrm{mg}$ of pegbovigrastim $7 \mathrm{~d}$ before expected calving and a second injection within $24 \mathrm{~h}$ of calving. Vaginal samples from 97 PEG-treated and 98 CTR cows were collected at calving, $7 \pm 3$, and $35 \pm 3 \mathrm{~d}$ in milk (0, 7, and 35 DIM). Metritis was diagnosed at $7 \pm 3$ DIM and purulent vaginal discharge (PVD) at $35 \pm 3$ DIM. The PEG treatment did not alter the vaginal microbiome. Principal coordinate analysis (PCoA) showed that metritic cows had a dissimilar vaginal microbiome compared with cows that did not develop metritis, particularly at 7 but also at 35 DIM. This difference was characterized by higher relative abundance of Porphyromonas and Bacteroides and a lower relative abundance of Ureaplasma, Ruminococcaceae, and Clostridiales at 7 DIM, and a higher relative abundance of Ureaplasma and a lower relative abundance of Pasteurellaceae at 35 DIM. Based on PCoA, we observed that cows that developed PVD had a dissimilar vaginal microbiome compared with cows that did not develop PVD, particularly at 35 DIM but also at 7 DIM. This difference was characterized by a higher relative abundance of Bacteroides at 7 DIM and higher relative abundance of Fusobacterium and Bacteroides at 35 DIM. Cows that developed metritis and PVD also had higher relative abundance of Fusobacterium and Bacteroides at 0 DIM. Furthermore, the Chao1 and Shannon indices were decreased in metritic

Received October 1, 2018.

Accepted December 15, 2018.

*Corresponding author: rcb28@cornell.edu cows at 7 DIM and in PVD cows at 7 and 35 DIM. In summary, PEG treatment had no effect on the vaginal microbiome, and uterine disease was associated with major changes in the microbiome found in the vagina postpartum.

Key words: pegbovigrastim, microbiome, mastitis, metritis

\section{INTRODUCTION}

Improvements in genetics, housing, nutrition, and management have resulted in dramatic increases in milk production in the last $70 \mathrm{yr}$, from $\sim 2,000 \mathrm{~kg} / \mathrm{yr}$ in 1944 to over 10,000 kg/yr in 2016 (Capper et al., 2009; Santos et al., 2010; USDA-NASS, 2017). Nonetheless, the improvements in milk production have not been accompanied by improvements in health. Approximately $45 \%$ of dairy cows experience a disease or disorder in the first $60 \mathrm{~d}$ postpartum (Santos et al., 2010), and metritis and mastitis are two of the most prevalent and costly diseases to the dairy industry (Goshen and Shpigel, 2006; Overton and Fetrow, 2008; Cha et al., 2011).

Innate immunity is the most important defense system of the bovine uterus and mammary gland, and the neutrophil is the main cell type involved in innate immunity in those organs (Hussain, 1989; Paape et al., 2003). However, during the transition into lactation, dairy cows experience a reduction in leukocyte function, including a reduction in neutrophil phagocytosis and killing capacity, which is believed to be a predisposing factor to infectious diseases such as metritis and mastitis (Goff and Horst, 1997; Hammon et al., 2006; Galvão and Santos, 2014).

Granulocyte colony-stimulating factors (G-CSF) are cell-signaling glycoproteins involved in hematopoiesis and inflammation (Nicola et al., 1983; Souza et al., 1986; Zsebo et al., 1986; Groopman et al., 1989). Nickerson et al. (1989) first identified a role for G-CSF in prevention of clinical mastitis. They observed that treatment with recombinant G-CSF led to an increase in circulating neutrophils, an increase in migration of neutrophils to 
the mammary gland after an experimental challenge with Staphylococcus aureus, and a numerical reduction in new infections in challenged quarters (Nickerson et al., 1989). Later, Kimura et al. (2014) showed that treatment of periparturient dairy cows with pegylated recombinant bovine G-CSF (pegbovigrastim, Imrestor, Elanco Animal Health, Greenfield, IN) led to a dramatic increase in the number of circulating neutrophils and an increase in myeloperoxidase release after stimulation in vitro, which indicated a possible increase in neutrophil extracellular killing ability (Kimura et al., 2014).

The administration of pegbovigrastim (PEG) $7 \mathrm{~d}$ before expected calving and on the day of calving reduced the number of naturally occurring clinical mastitis cases (Hassfurther et al., 2015; Ruiz et al., 2017). However, in a recent study conducted by our group, PEG treatment did not prevent the occurrence of clinical mastitis (Zinicola et al., 2018). It has been reported that PEG treatment resulted in a small but significant increase in the incidence of metritis, although the negative effects of metritis on milk yield were less severe in multiparous PEG-treated cows that developed metritis (Ruiz et al., 2017). Similar results on milk yield in multiparous cows diagnosed with metritis and treated with PEG were observed (Zinicola et al., 2018); however, in that study, the administration of PEG did not alter the incidence of metritis.

Recently, we observed that metritic cows treated with PEG had a higher proportion of neutrophils in the vagina at 7 DIM compared with metritic control cows (Zinicola et al., 2018). In addition, we observed a greater reduction in blood neutrophils in PEG-treated cows diagnosed with metritis compared with metritic control cows, indicating that more neutrophils were migrating to the reproductive tract in PEG-treated cows (Zinicola et al., 2018). Neutrophil migration and function is believed to be critical for prevention or elimination of infection in the reproductive tract. Thus, one way to better understand the effects of PEG on disease establishment in the reproductive tract is to study the microbiome. We have shown that metritis is associated with a dysbiosis of the normal uterine microbial flora (Jeon et al., 2015). This dysbiosis is characterized by an increased in the relative abundance of gram-negative anaerobes such as Bacteroidetes and Fusobacteria and a decrease in the relative abundance of Firmicutes (Jeon et al., 2015). Therefore, we hypothesized that periparturient dairy cattle treated with PEG would have a greater migration of neutrophils to the uterus, which would prevent the microbial dysbiosis associated with metritis. Therefore, our primary objective was to characterize the microbiota found in the vagina postpartum in cows treated with PEG $7 \mathrm{~d}$ from the expected calving date and within $24 \mathrm{~h}$ of calving. A secondary objective was to compare the microbiome in the vagina postpartum in cows that developed uterine disease with that of cows that did not develop uterine disease.

\section{MATERIALS AND METHODS}

\section{Cows, Housing, and Feeding}

The study was conducted in a large commercial dairy farm located near Ithaca, New York, from September 2016 to December 2016. All animal procedures were approved by the Cornell University Institutional Animal Care and Use Committee (protocol number 2016-0028). The farm milked $\sim 3,800$ Holstein cows 3 times daily in a rotary parlor with integrated milk meters that record individual production at every milking (DeLaval, Tumba, Sweden). The cows were housed in freestall barns, with concrete stalls bedded with composted manure solids treated with quicklime $(\mathrm{CaO})$. After calving, all cows were offered a TMR with feed pushed up 6 to 8 times a day and consisting of approximately $55 \%$ forage (corn silage, haylage, and wheat straw) and $45 \%$ concentrate (corn meal, soybean meal, canola, cottonseed, and citrus pulp) on a DM basis. The diet was formulated to meet or exceed National Research Council nutrient requirements for lactating Holstein cows weighing $650 \mathrm{~kg}$ and producing $45 \mathrm{~kg}$ of $3.5 \%$ FCM (NRC, 2001). As cows demonstrated signs of calving, they were moved to individual maternity pens for delivery, where trained farm personnel assisted with parturition as needed. Calves were removed from their dams immediately after birth.

\section{Study Cows}

The cows used for this study are a subset of cows enrolled in a larger experiment that evaluated the effect of PEG on periparturient diseases, milk production, and reproductive performance of Holstein cows (Zinicola et al., 2018). For the larger experiment, 840 cows were randomly assigned within parity group (primigravid or multigravid) to 1 of 2 treatments using a treatment allocation sheet built using the random number generator formula in Excel (Microsoft Corp., Redmond, WA): PEG $(\mathrm{n}=417)$ or untreated control $(\mathbf{C T R} ; \mathrm{n}=$ 423). The PEG-treated cows received a subcutaneous injection containing $15 \mathrm{mg}$ of pegbovigrastim in $2.7-\mathrm{mL}$ prefilled syringes (Imrestor, Elanco Animal Health) $7 \mathrm{~d}$ before expected calving and a second injection within 24 $\mathrm{h}$ of calving. The CTR group did not receive any treatment. Treatments were administered by our research group. To avoid biases, treatment identification was not listed in the data collection forms or in the on-farm herd management software program. A subset of PEG $(\mathrm{n}=$ 
102; 37 primiparous and 65 multiparous) and CTR (n $=103 ; 39$ primiparous and 64 multiparous) cows was randomly selected (every fourth cow enrolled) to evaluate the reproductive tract microbiome postpartum by collecting swab samples from the vagina at $0,7 \pm 3$, and $35 \pm 3$ DIM. Five vaginal swabs were not collected from each of the treatment groups in multiparous cows. Therefore, a subset of 97 PEG-treated (37 primiparous and 60 multiparous) and 98 CTR cows (39 primiparous and 59 multiparous) cows were sampled for the evaluation of the microbiome found in the vagina postpartum.

\section{Sample Collection and DNA Extraction}

Vaginal discharge was sampled from the caudal portion of the vagina by a member of the research team at calving (hereafter 0 DIM), $7 \pm 3$, and $35 \pm 3$ DIM ( $\mathrm{n}=$ 580) using individually wrapped, sterile, cotton-tipped swabs as previously reported (Bicalho et al., 2017a). Samples were collected immediately before evaluation of vaginal discharge using the Metricheck device (Simcro, Hamilton, New Zealand). Briefly, the vulva was cleaned with alcohol wipes, the vulvar lips were opened, and the swab was introduced approximately $7.5 \mathrm{~cm}$ into the vagina, where the swab was rolled against the ventral portion of the vagina to sample the discharge draining from the uterus. The swabs were placed in sterile tubes, and the tubes were placed on ice and transported to the laboratory within $4 \mathrm{~h}$, where the swabs were stored at $-20^{\circ} \mathrm{C}$ until further processing. Metagenomic DNA was isolated from the swab samples using the BiOstic Bacteremia DNA Isolation Kit (Mo Bio Laboratories Inc., Carlsbad, CA) following the manufacturer's recommendations.

\section{Health Disorders Evaluated}

Metritis was diagnosed by a member of the research team at $7 \pm 3$ DIM by evaluating the discharge recovered from the fundus of the vagina using the Metricheck device as previously reported (Jeon et al., 2015). The discharge was scored using a 5-point scale (1: not fetid, normal lochia, viscous, clear, red, or brown; 2: cloudy, pink, red, or brown mucoid discharge with flecks of pus; 3: not fetid, pink red or brown mucopurulent discharge with $<50 \%$ pus; 4 : not fetid, pink, red, or brown purulent discharge with $\geq 50 \%$ pus; 5 : fetid red-brownish, watery discharge). Cows with a discharge score of 5 were classified as having clinical metritis. Farm personnel performed their own diagnosis and treatment of metritis at $7 \pm 3$ DIM (hereafter 7 DIM); however, coordination with the research team ensured cows were sampled before administration of antibiotics.
Purulent vaginal discharge (PVD) was diagnosed by a member of the research team at $35 \pm 3$ DIM (hereafter 35 DIM) by evaluating the discharge recovered from the fundus of the vagina using the Metricheck device. The discharge was scored using a 0 to 3 scale (0: no secretion material retrieved and/or clear mucus, 1: flecks of purulent material within otherwise clear mucus; 2 : $\leq 50 \%$ purulent material in the vaginal discharge, 3 : $>50 \%$ purulent material with/without fetid discharge); cows with a score of $\geq 2$ were diagnosed with PVD and cows with a score $\leq 1$ were classified as not having PVD (LeBlanc et al., 2002).

\section{S rRNA Gene Amplification and Sequencing}

The concentration and purity of DNA for all samples were evaluated using NanoDrop ND-1000 spectrophotometer (NanoDrop Technologies, Rockland, DE) at wavelengths of 260 and $280 \mathrm{~nm}$; the optical density ratio at 260/280 nm of DNA ranged between 1.7 and 2.0. For each metagenomic DNA sample, the V4 hypervariable region of the bacterial/archaeal $16 \mathrm{~S}$ rRNA gene was amplified using the $515 \mathrm{~F}$ and $806 \mathrm{R}$ primer set according to previously described methods and optimized for the Illumina MiSeq platform (Caporaso et al., 2012). The PCR products were tagged with 280 different 12-bp error-correcting Golay barcodes (http:/ /www.earthmicrobiome.org/; Gilbert et al., 2010). The $\mathrm{PCR}$ was carried out in triplicate in $25-\mu \mathrm{L}$ reactions using 12 to $300 \mathrm{ng}$ of template DNA, 1× EconoTaq Plus Green Master Mix (Lucigen, Middleton, WI), and 10 $\mu \mathrm{mol}$ of each primer. Thermal cycling consisted of an initial denaturing step of $94^{\circ} \mathrm{C}$ for 3 min followed by 35 cycles of $94^{\circ} \mathrm{C}$ for $45 \mathrm{~s}, 50^{\circ} \mathrm{C}$ for $1 \mathrm{~min}$, and $72^{\circ} \mathrm{C}$ for 90 $\mathrm{s}$, and a final elongation step of $72^{\circ} \mathrm{C}$ for $10 \mathrm{~min}$. Replicate amplicons were visualized on a $1.2 \%$ agarose gel stained with $0.5 \mathrm{mg} / \mathrm{mL}$ ethidium bromide, and then pooled and purified using the Gel/PCR DNA Fragment Extraction kit (IBI Scientific, Peosta, IA).

The amplicon DNA concentration in each sample was measured using a NanoDrop ND-1000 spectrophotometer (NanoDrop Technologies) at wavelengths of 260 and $280 \mathrm{~nm}$. Amplicons were standardized to the same concentration and pooled into 3 sequencing runs according to individual barcode primers for the $16 \mathrm{~S}$ rRNA gene. Final equimolar libraries were sequenced using the MiSeq reagent kit v2 (300 cycles) on the MiSeq platform (Illumina Inc., San Diego, CA). The generated 16S rRNA gene sequences were processed through the open source software pipeline Quantitative Insights into Microbial Ecology (QIIME; http://qiime .org/; version 1.9.0) as previously reported (Caporaso et al., 2010; Bicalho et al., 2017a). Sequences were fil- 
tered for quality using established guidelines (Bokulich et al., 2013). Sequences were binned into operational taxonomic units (OTU) based on 97\% identity using UCLUST (Edgar, 2010) against the Greengenes reference database (McDonald et al., 2012). Low-abundance clusters were filtered and chimeric sequences were removed using USEARCH (Edgar, 2010). We compared the representative sequences for each OTU against the Greengenes database for taxonomy assignment and used only full-length, high-quality reads $(\mathrm{r}=3)$ for analysis, where $r$ is the minimum number of consecutive bases with Q-scores lower than q from the beginning of the read to identify the location of quality trimming and $\mathrm{q}$ is the Q-score threshold that classifies a base as low quality. Illumina sequencing of the $\mathrm{V} 4$ region of the $16 \mathrm{~S}$ rRNA gene provides excellent sequencing depth and coverage, but the shorter reads $(\sim 300 \mathrm{bp})$ may provide inaccurate species classification, especially for species with high 16S rRNA gene similarity such as Escherichia and Shigella species; therefore, only classification to the genus level was attempted and presented (Chakravorty et al., 2007).

\section{Data Analysis}

Samples from the vagina resulted in 21,411,210 highquality reads after filtering for size, quality, and the presence of chimeras. Four vaginal samples, 1 from PEG on d 0, 2 from PEG on d 7, and 1 from CTR on d 7 , did not pass the filtering criteria and were excluded from the study. One CTR cow died before metritis was evaluated, and 3 PEG-treated cows and 5 CTR cows died or were sold before PVD was evaluated. Therefore, 97 PEG-treated and 97 CTR cows were available for metritis analysis and only 94 PEG-treated and 93 CTR cows were available for PVD analysis.

Principal coordinates analysis (PCoA) of BrayCurtis dissimilarity was used to compare treatment groups, time points, and health categories (e.g., metritis vs. no metritis) using the lowest taxonomic classification available using PAST version 3.18 (https:/ /palaeo-electronica.org/2001_1/past/issue1_01.htm). The effects of treatment, health category, time $(0,7,35$ DIM), and the interaction between treatment or health category and time on the microbiome structure found in the vagina were evaluated with 2-way permutational multivariate ANOVA (PERMANOVA) with 9,999 permutations based on Bray-Curtis dissimilarity using the lowest taxonomic classification available using PAST version 3.18. The effect of antibiotic treatment of metritis, time (7 and 35 DIM), and the interaction between antibiotic treatment and time on the microbiome structure found in the vagina of cows that developed metritis was evaluated with 2-way PERMANOVA with 9,999 permutations based on Bray-Curtis dissimilarity using the lowest taxonomic classification. Continuous outcomes such as the relative abundances of bacterial taxa, Chao1 richness index, and Shannon diversity index were compared using ANOVA for repeated measures for vaginal samples using the MIXED procedure of SAS (version 9.4; SAS Institute Inc., Cary, NC). Normality and homoscedasticity of residuals were assessed using residual plots. For repeated measures, unstructured, autoregressive 1, and compound symmetry covariance structures were tested, and the one with the smallest Akaike information criterion was chosen. For all models, Tukey-Kramer adjustment for multiple comparisons was performed.

Because PEG has been shown to cause a slight increase in the incidence of metritis (Ruiz et al., 2017), we evaluated the effect of PEG treatment without including uterine disease as an independent variable. The independent variables offered to the ANOVA model were treatment (PEG vs. CTR), time (0, 7, 35 DIM), parity (primiparous vs. multiparous), and 2-way interactions between treatment and other variables. Then, we evaluated the association between the vaginal microbiome and the development of uterine disease postpartum controlling for any effect of treatment in 2 separate models. Two models were run for each uterine disease because metritis is correlated with PVD (Spearman rho $=0.22 ; P<0.001)$ and because there were cows with missing diagnoses of metritis or PVD. The independent variables offered to the ANOVA models were treatment (PEG vs. CTR), time (0, 7, 35 DIM), parity (primiparous vs. multiparous), uterine disease (metritis vs. no metritis, PVD vs. no PVD), and 2-way interactions between variables.

The effect of PEG treatment on uterine disease was evaluated using logistic regression, and the model included the fixed effects of treatment (PEG vs. CTR) and parity (primiparous vs. multiparous). For all models, the effect of treatment was forced in, but other variables were removed according to the Wald statistics criterion when $P>0.10$. Significance was considered when $P \leq 0.05$.

\section{RESULTS}

\section{Descriptive Data}

Twenty-six cows (13.4\%) were diagnosed with metritis, and 25 cows $(13.4 \%)$ were diagnosed with PVD. Of the 26 cows diagnosed with metritis by our research team, only $12(46.2 \%)$ were treated with antibiotics by the farm personnel. 


\section{Comparison of Microbiome in the Vagina Between Treatment Groups over Time}

Microbial community richness and diversity postpartum were analyzed using the Chao1 and Shannon indices, respectively. We detected no effect of treatment $(P$ $>0.42)$ or interaction between treatment and time $(P$ $>0.65$ ) on richness or diversity indices (Figure 1A, 1B). There was an effect of time on richness and diversity $(P$ $<0.01)$. The Chao1 richness index was significantly $(P$ $<0.01$ ) lower at 7 than at 0 and 35 DIM (Figure 1A). The Shannon diversity index was significantly higher $(P<0.05)$ at 0 than at 7 DIM (Figure 1B), and at 35 than at 0 and 7 DIM (Figure 1B).
We used PCoA paired with PERMANOVA based on the Bray-Curtis dissimilarity of relative abundance using the lowest taxonomic classification available to evaluate the effect of PEG treatment, time, and the interaction between treatment and time on the structure of the microbiome found in the vagina postpartum. There was no effect of PEG treatment $(P=0.47)$ or interaction between treatment and time $(P=0.97)$. For ease of visualization, the effect of treatment on the microbiome structure found in the vagina postpartum is presented separately at 0,7 , and 35 DIM (Figures $2 \mathrm{~A}, 2 \mathrm{~B}$, and 2C). Despite not finding a significant effect of PEG treatment on the microbiome structure, we compared the relative abundance of the 15 most
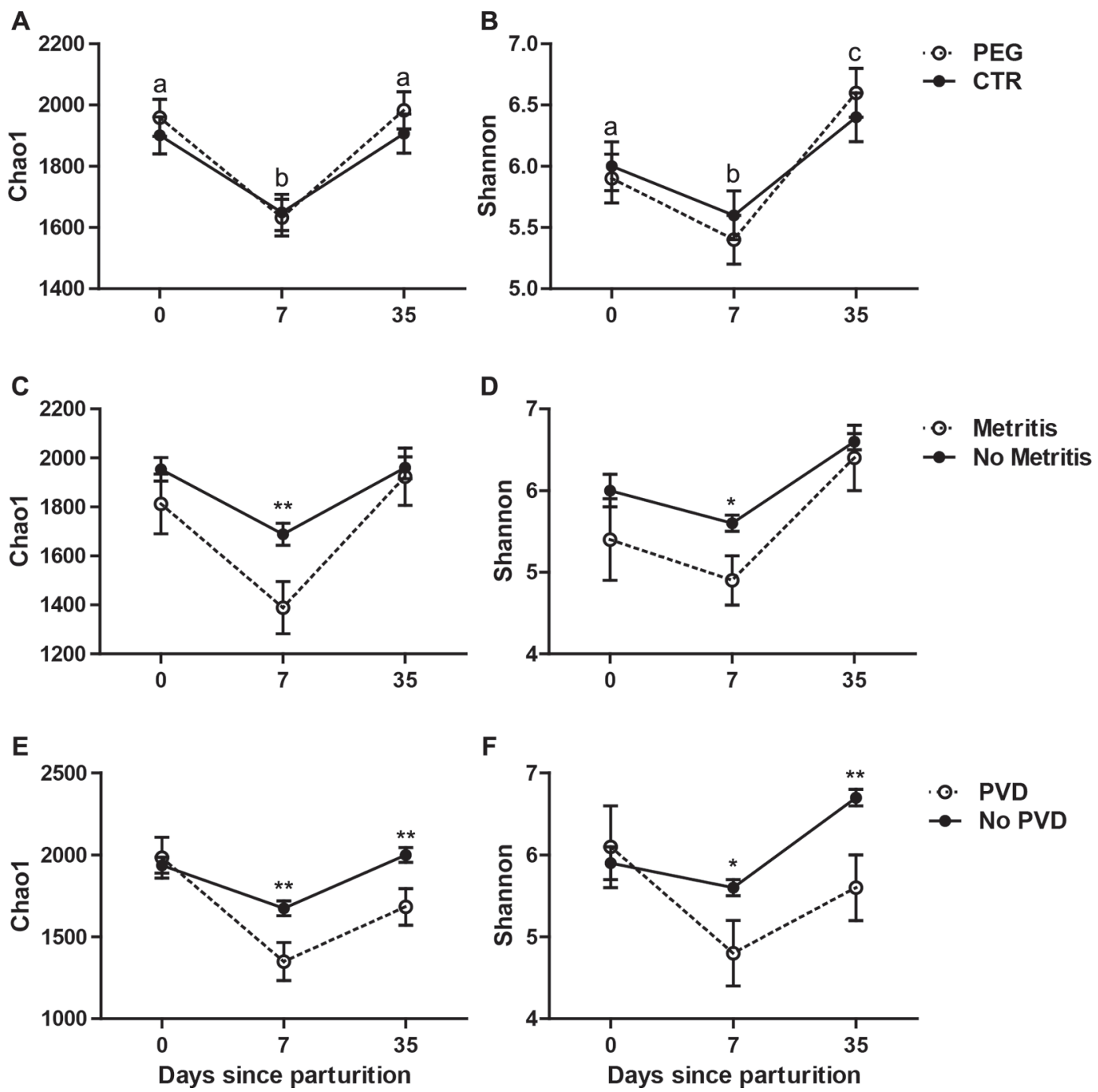

Figure 1. Diversity indices for the reproductive tract microbiota at 0, 7, and 35 DIM. (A) Chao1 richness and (B) Shannon diversity indices for cows treated (PEG) and untreated (control, CTR) with pegbovigrastim. Pegbovigrastim-treated cows received a subcutaneous injection containing $15 \mathrm{mg}$ of pegbovigrastim in 2.7-mL prefilled syringes (Imrestor, Elanco Animal Health, Greenfield, IN) $7 \mathrm{~d}$ before expected calving and a second injection within $24 \mathrm{~h}$ of calving. (C, E) Chao1 richness and (D, F) Shannon diversity indices for cows diagnosed with and without metritis at 7 DIM $(\mathrm{C}, \mathrm{D})$ or with and without purulent vaginal discharge (PVD) at 35 DIM (E, F). Letters $(\mathrm{a}, \mathrm{b}, \mathrm{c})$ indicate significant $(P \leq$ $0.05)$ differences between time points; *significant difference $(P \leq 0.05)$ between groups within a time point; ** significant $(P \leq 0.01)$ difference between groups. Error bars represent SEM. 
abundant taxa found in all vaginal samples between treatments. These taxa had an average prevalence $\geq 2 \%$ and represented $65 \%$ of all assigned taxa. Likewise, there was no effect of PEG treatment $(P>0.10)$ or interaction between treatment and time $(P>0.15)$ on the 15 most abundant taxa found in all vaginal samples (Figure 3A). Finally, we evaluated the effect of PEG treatment on the relative abundance of the 6 most prevalent phyla in all vaginal samples: Firmicutes, Bacteroidetes, Fusobacteria, Proteobacteria, Tenericutes, and Actinobacteria. These phyla had an average prevalence $\geq 2 \%$ and represented $98 \%$ of all phyla. The remaining phyla were combined into a single category termed "others." There was no effect of PEG treatment $(P>0.25)$ or interaction between treatment and time $(P>0.50)$ on the 6 most abundant phyla or "others" in vaginal samples (Supplemental Figure S1A; https://doi .org/10.3168/jds.2018-15783).

We detected an effect of time $(P<0.01)$ on the microbiome structure (Figure 2D). The microbiome structure was significantly different between d 0 and 7 $(P<0.01)$, d 0 and $35(P<0.01)$, and $\mathrm{d} 7$ and $35(P$ $<0.01)$. Comparison of the relative abundance of the 15 most abundant taxa found in all vaginal samples between time points revealed an effect of time $(P<0.01)$ on the relative abundance of family Ruminococcaceae, genus Streptococcus, genus Fusobacterium, genus Porphyromonas, order Clostridiales, genus 5-7N15, order Bacteroidales, family Leptotrichiaceae, genus Aggregatibacter, genus Helcococcus, family Lachnospiraceae, genus Corynebacterium, and genus Bacteroides (Figure 3B). There was also a significant effect of time $(P<$ 0.05) on the relative abundance of Firmicutes, Bacteroidetes, Proteobacteria, Fusobacteria, Tenericutes, Actinobacteria, and "others" (Supplemental Figure S1A; https://doi.org/10.3168/jds.2018-15783).

\section{Comparison of the Microbiome Found in the Vagina Between Cows that Did and Did Not Develop Uterine Disease}

The Chao1 richness index of the microbiome in the vagina was decreased in cows that developed metritis postpartum compared with cows that did not $(1,708.4$ \pm 75.0 vs. $1,867.1 \pm 29.8 ; P=0.05)$, mostly because of a significant decrease in metritic cows at 7 DIM $(1,388.8 \pm 106.8$ vs. $1,688.4 \pm 44.9 ; P=0.01$; Figure $1 \mathrm{C})$. The Shannon diversity index of the microbiome found in the vagina was also decreased in cows that developed metritis postpartum compared with those that did not ( $5.5 \pm 0.3$ vs. $6.1 \pm 0.1 ; P=0.05)$, mostly because of a significant decrease in metritic cows at 7 DIM (4.9 \pm 0.3 vs. $5.6 \pm 0.1 ; P=0.05$; Figure 1D). There was no interaction $(P>0.50)$ between PEG treatment and metritis development on the Chao1 and Shannon indices.

By using PCoA paired with PERMANOVA, we observed that the vaginal microbiome structure was different $(P<0.01)$ in cows that did and did not develop metritis, mostly because of a significant difference at 7 DIM $(P<0.01$; Figure $4 \mathrm{~B})$ but also at 35 DIM $(P$ $=0.03$; Figure $4 \mathrm{C}$ ). There was no association between antibiotic treatment of metritis at 7 DIM and vaginal microbiome structure at 7 and 35 DIM, as evidenced by the lack of an effect of antibiotic treatment $(P=0.07)$ and the lack of an interaction between antibiotic treatment and time $(P=0.24)$. Comparison of the relative abundance of the 15 most abundant taxa found in all vaginal samples between cows that did and did not develop metritis showed that cows that developed metritis had higher relative abundance of Fusobacterium $(9.0 \pm$ 1.2 vs. $6.2 \pm 0.5 ; P=0.04)$, Porphyromonas $(7.8 \pm 1.1$ vs. $4.4 \pm 0.4 ; P<0.01)$, Bacteroides (5.6 \pm 0.6 vs. 1.4
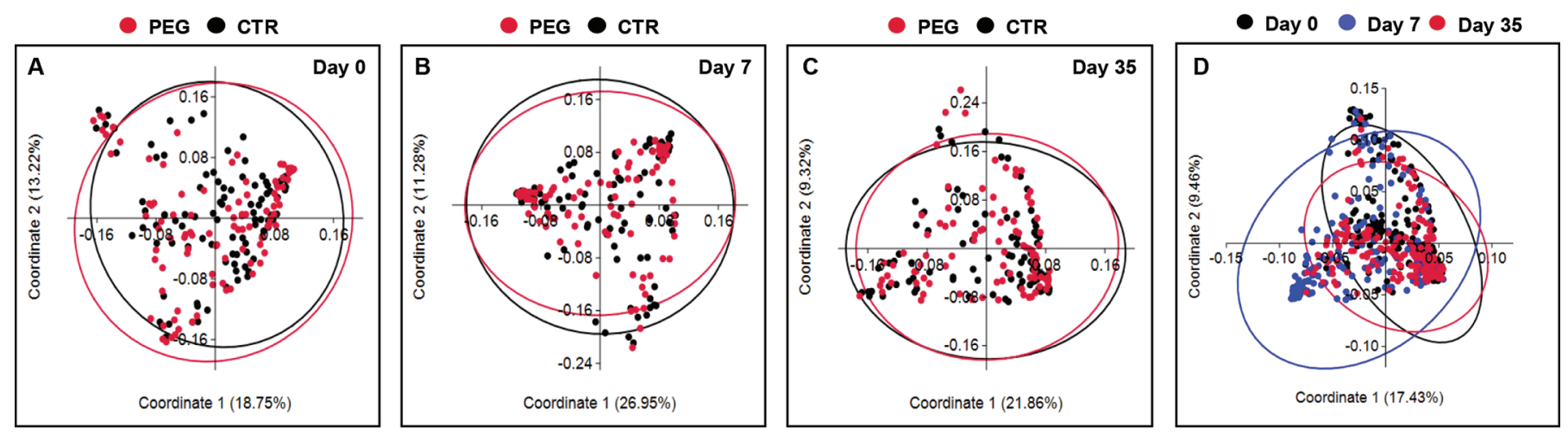

Figure 2. Assessment of the effect of pegbovigrastim treatment (PEG) on the reproductive tract microbiome using principal coordinate analysis of Bray-Curtis dissimilarity using the lowest taxonomic classification available at 0 (A), 7 (B) and 35 (C) DIM, and the assessment of the effect of time on the reproductive tract microbiome (D). There was no effect of treatment $(P>0.35)$, but there was an effect of time $(P<$ $0.001)$ on the reproductive tract microbiome. CTR $=$ control. 
$\pm 0.2 ; P<0.01)$, and lower relative abundance of genus $5-7 N 15$ (Bacteroidaceae family; $2.0 \pm 0.6$ vs. $2.8 \pm 0.1$; $P=0.02)$. Interactions between metritis and time $(P$ $\leq 0.05$ ) also showed that cows that developed metritis had higher relative abundance of Fusobacterium and Bacteroides at 0 DIM, higher relative abundance of Porphyromonas and Bacteroides at 7 DIM, and higher relative abundance of Ureaplasma at 35 DIM (15.7 \pm 3.0 vs. $6.1 \pm 1.2 ; P<0.01)$ compared with cows that did not develop metritis (Figure 5A, 5B, 5C). In contrast, cows that developed metritis had lower relative abundance of Ureaplasma $(3.8 \pm 3.9$ vs. $12.2 \pm 1.6 ; P$ $=0.05)$, Ruminococcaceae $(3.3 \pm 1.4$ vs. $6.5 \pm 0.6 ; P=$ $0.03)$ and Clostridiales at 7 DIM (1.2 \pm 0.6 vs. $2.8 \pm$ $0.2 ; P=0.01)$, and lower abundance of Pasteurellaceae at 35 DIM $(0.7 \pm 2.0$ vs. $5.8 \pm 0.8 ; P=0.02)$ compared with cows that did not develop metritis (Figure $5 \mathrm{~A}$, 5B, 5C). Phylum-level data were evaluated, and cows that developed metritis had higher relative abundance of Bacteroidetes compared with cows that did not develop metritis $(21.0 \pm 1.6$ vs. $15.8 \pm 0.6 ; P<0.01)$. Interactions between metritis and time $(P \leq 0.05)$ also showed that cows that developed metritis had higher relative abundance of Bacteroidetes at 7 DIM (39.1 \pm 3.6 vs. $20.5 \pm 1.5 ; P<0.01)$, and higher relative abundance of Tenericutes $(18.2 \pm 3.1$ vs. $7.1 \pm 1.2 ; P$ $<0.01)$, Actinobacteria $(8.6 \pm 1.6$ vs. $5.1 \pm 0.6 ; P=$ $0.04)$, and "others" at 35 DIM ( $3.7 \pm 0.5$ vs. $2.3 \pm 0.2$; $P=0.02)$ compared with cows that did not develop metritis (Supplemental Figure S2B, S2C; https://doi
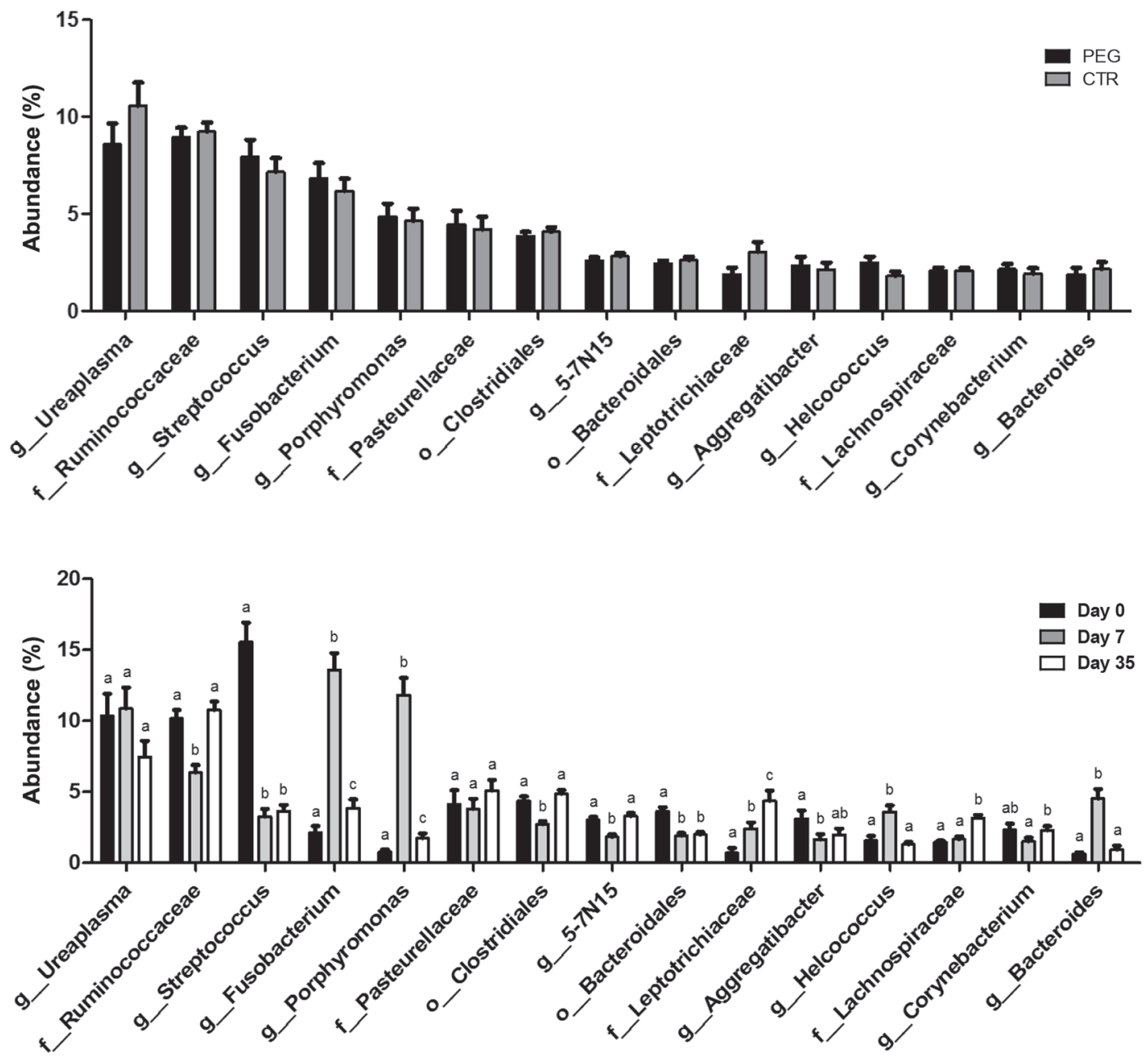

Figure 3. Overall mean relative abundance of the 15 most prevalent bacterial taxa (letters preceding name indicate taxon: $\mathrm{g}=\mathrm{genus}, \mathrm{f}=$ family, $o=$ order) found in the reproductive tract at 0,7 and 35 DIM in cows treated (PEG) and untreated (CTR) with pegbovigrastim (top panel), and the mean relative abundance of the 15 most prevalent bacterial taxa found in the reproductive tract at 0 , 7 and 35 DIM (bottom panel). Pegbovigrastim-treated cows received a subcutaneous injection containing $15 \mathrm{mg}$ of pegbovigrastim in 2.7-mL prefilled syringes (Imrestor, Elanco Animal Health, Greenfield, IN) $7 \mathrm{~d}$ before expected calving and a second injection within $24 \mathrm{~h}$ of calving. Treatment had no effect $(P>0.10)$ on the most prevalent bacterial taxa. Letters $(\mathrm{a}, \mathrm{b}, \mathrm{c})$ indicate significant $(P \leq 0.05)$ differences between time points. Error bars represent SEM. 
Metritis

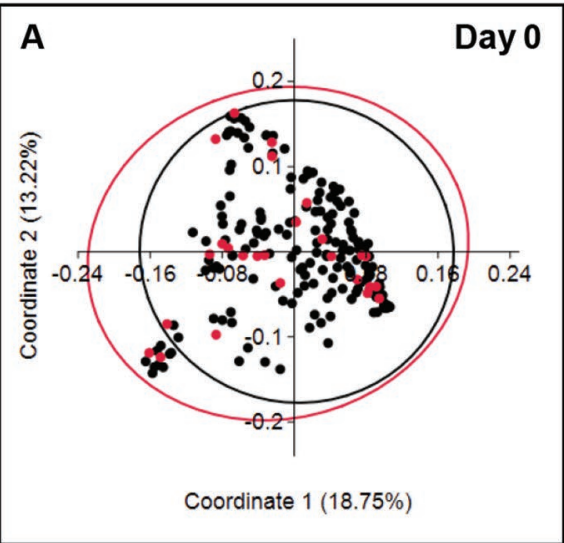

Metritis

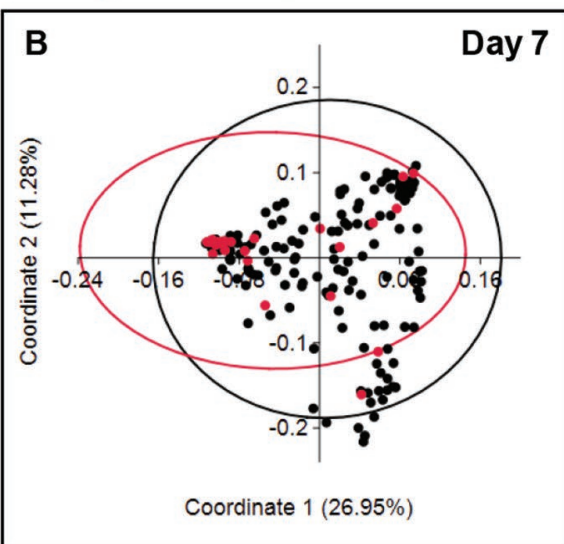

Metritis

No Metritis

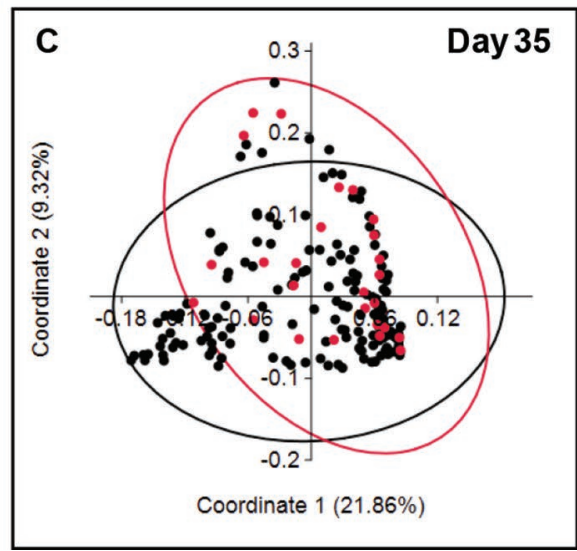

Figure 4. Evaluation of the association between the reproductive tract microbiome at 0 (A), 7 (B) and 35 (C) DIM and the diagnosis of metritis at 7 DIM using principal coordinate analysis of Bray-Curtis dissimilarity using the lowest taxonomic classification available. There was no association between metritis diagnosis at 7 DIM and the reproductive tract microbiome at 0 DIM $(P=0.12)$, but there was an association between metritis diagnosis at 7 DIM and the reproductive tract microbiome at $7(P<0.001)$ and $35(P=0.03)$ DIM.

.org/10.3168/jds.2018-15783). In contrast, cows that developed metritis had lower relative abundance of Firmicutes $(22.9 \pm 3.6$ vs. $31.4 \pm 1.5 ; P=0.03)$, Tenericutes $(5.0 \pm 4.1$ vs. $14.9 \pm 1.6 ; P=0.03)$, and "others" at 7 DIM $(0.6 \pm 0.3$ vs. $1.4 \pm 0.1 ; P=0.02)$, and lower relative abundance of Proteobacteria at 35 DIM (4.5 \pm 3.2 vs. $17.3 \pm 1.3 ; P<0.01$; Supplemental Figure S2B, S2C; https://doi.org/10.3168/jds.2018-15783). There was no interaction $(P>0.15)$ between PEG treatment and metritis on the relative abundance of the 15 most abundant taxa or the 6 most abundant phyla.

The Chao1 richness index of the microbiome found in the vagina was decreased in cows that developed PVD compared with cows that did not $(1,671.7 \pm 76.6$ vs. $1,870.6 \pm 30.4 ; P=0.02)$, mostly because of a significant decrease in cows with PVD at 7 DIM (1,349.4 \pm 116.0 vs. $1,674.3 \pm 45.6 ; P=0.01)$ and $35 \mathrm{DIM}$ $(1,682.6 \pm 111.9$ vs. $2,000.1 \pm 44.9 ; P<0.01$; Figure $1 \mathrm{E})$. The Shannon diversity index of the microbiome found in the vagina was also decreased in cows that developed PVD postpartum compared with cows that did not $(5.5 \pm 0.3$ vs. $6.1 \pm 0.1 ; P=0.04)$, mostly because of a significant decrease in cows with PVD at 7 DIM (4.8 \pm 0.4 vs. $5.6 \pm 0.1 ; P=0.04)$ and 35 DIM $(5.6 \pm 0.4$ vs. $6.7 \pm 0.1 ; P<0.01$; Figure $1 \mathrm{~F})$. There was no interaction $(P>0.35)$ between PEG treatment and PVD on the Chao1 and Shannon indices.

By using PCoA paired with PERMANOVA, we observed that the vaginal microbiome structure was different $(P<0.01)$ in cows that did and did not develop PVD, mostly because of a significant difference at 7 DIM $(P=0.03$; Figure $6 \mathrm{~B})$ and $35 \operatorname{DIM}(P=0.02$; Figure $6 \mathrm{C})$. Comparison of the relative abundance of the 15 most abundant taxa found in all vaginal sam- ples between cows that did and did not develop PVD showed that cows that developed PVD had higher relative abundances of Fusobacterium (10.9 \pm 1.2 vs. 5.9 $\pm 0.5 ; P=0.04)$ and Bacteroides (5.0 \pm 0.7 vs. $1.6 \pm$ $0.3 ; P<0.01)$. Interactions between PVD and time $(P$ $\leq 0.05)$ showed that cows that developed PVD had significantly $(P<0.05)$ increased relative abundance of Fusobacterium only at 0 and 35 DIM, not at 7 DIM (Figure 7A, 7B, 7C) compared with cows that did not develop PVD. Phylum-level data showed that cows that developed PVD had higher relative abundance of Bacteroidetes compared with cows that did not develop PVD (20.1 \pm 16 vs. $16.2 \pm 0.7 ; P=0.03)$. Interactions between PVD and time $(P \leq 0.05)$ showed that cows that developed PVD had higher relative abundance of Fusobacteria at 0 DIM, higher relative abundance of Bacteroidetes at 7 DIM, and lower relative abundance of Firmicutes at 7 and 35 DIM (Supplemental Figure S3A, S3B, S3C; https://doi.org/10.3168/jds.2018 -15783). There was no interaction $(P>0.15)$ between PEG treatment and PVD on the relative abundance of the 15 most abundant taxa or the 6 most abundant phyla.

\section{DISCUSSION}

The use of PEG was previously shown to dramatically increase the number of neutrophils and their extracellular killing ability (Kimura et al., 2014; Zinicola et al., 2018). Nonetheless, PEG treatment results in a small but significant increase in the incidence of metritis (Ruiz et al., 2017). Little is known regarding the effect of PEG treatment on the microbiome of the postpartum reproductive tract. Therefore, in this study 
we assessed the microbiome found in the vagina in response to PEG treatment. We also investigated the association between the vaginal microbiome and the development of uterine disease, adjusting for any effect of treatment.

Treatment with PEG did not alter the structure or composition of the microbiome found in the vagina postpartum. We expected that a greater proportion of circulating neutrophils in PEG-treated cows would translate into a greater influx of neutrophils into the uterus shortly after calving, which would be expected to constrain the growth of gram-negative anaerobes such as Bacteroidetes and Fusobacteria (Jeon et al., 2015; Knudsen et al., 2016; Sicsic et al., 2018). Nonetheless, PEG treatment induced a reduction in circulating neutrophils combined with an increase in neutrophil counts
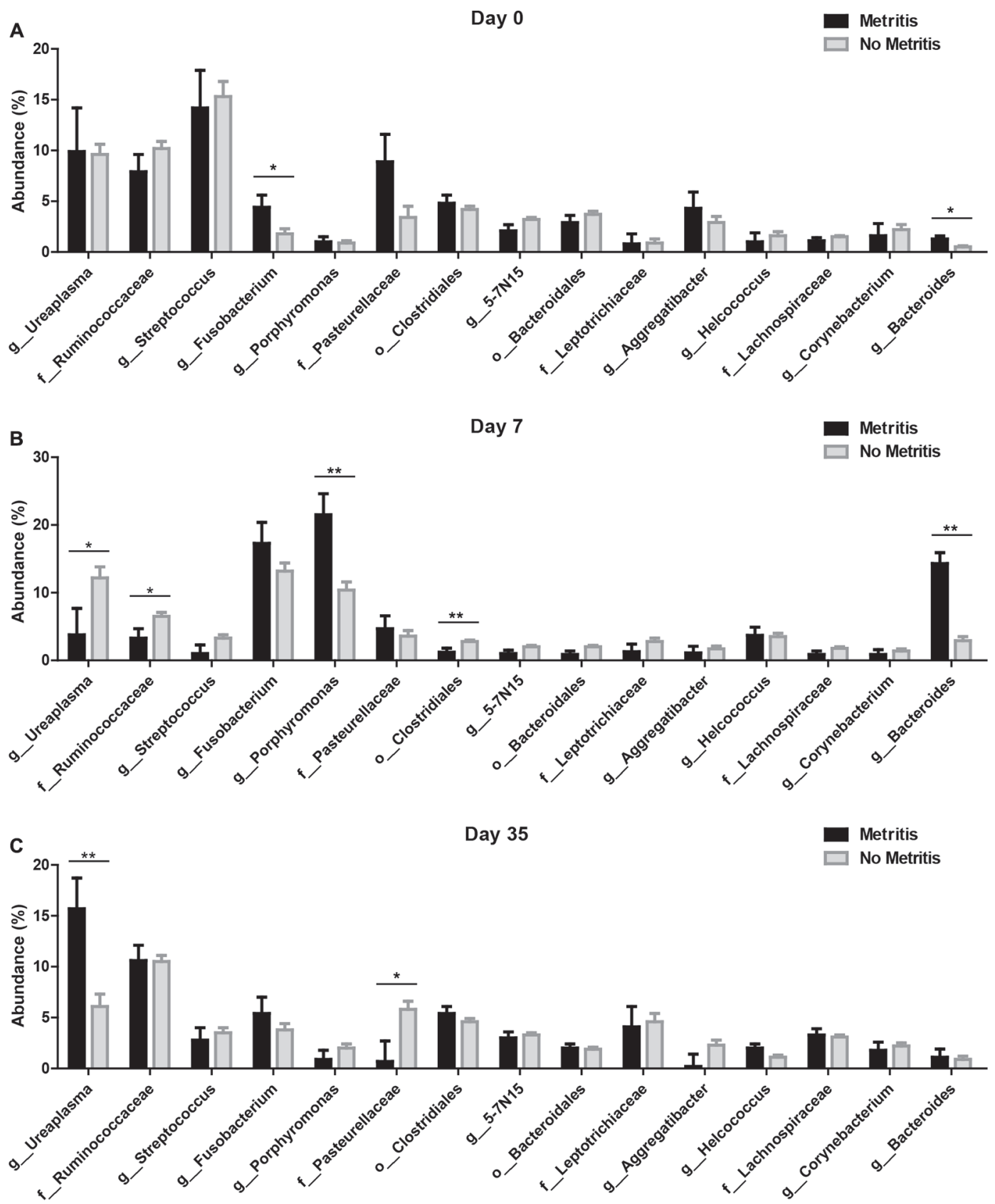

Figure 5. Mean relative abundance of the 15 most prevalent bacterial taxa (letters preceding name indicate taxon: $\mathrm{g}=$ genus, $\mathrm{f}=$ family, $\mathrm{o}=$ order $)$ found in the reproductive tract at $0(\mathrm{~A}), 7(\mathrm{~B})$ and $35(\mathrm{C})$ DIM in cows diagnosed or not with metritis at 7 DIM. *Significant $(P \leq$ $0.05)$ difference between groups; ** significant $(P \leq 0.01)$ difference between groups. Error bars represent SEM. 
in the vagina only in cows diagnosed with metritis and only at 7 DIM, not in all cows and not at calving (Zinicola et al., 2018). This finding indicates an increased influx of neutrophil in PEG-treated cows after uterine microbiota dysbiosis but not before; therefore, PEGtreatment seems ineffective at preventing the excessive growth of gram-negative anaerobes such as Bacteroidetes and Fusobacteria. This increased influx of neutrophils into the uterus after uterine microbiota dysbiosis and the associated inflammation caused by neutrophil degranulation and oxidative burst activity might lead to more pronounced clinical signs of metritis such as watery uterine discharge, which may lead to slightly more diagnoses of metritis in PEG-treated cows (Ruiz et al., 2017; Zinicola et al., 2018).

Herein, we saw that cows that developed metritis had an altered vaginal microbial structure and composition, particularly at 7 DIM but also at 35 DIM. Based on PCoA using the lowest taxonomic assignment, we saw that metritic cows had a dissimilar vaginal microbial structure and composition compared with cows that did not develop metritis at 7 and 35 DIM, which was characterized by higher relative abundance of Porphyromonas and Bacteroides and a lower relative abundance of Ureaplasma, Ruminococcaceae, and Clostridiales at 7 DIM, and a higher relative abundance of Ureaplasma and a lower relative abundance of Pasteurellaceae at 35 DIM. Although at calving, the PCoA analysis showed no differences between cows that later developed metritis and cows that did not, cows that developed metritis had higher relative abundances of Fusobacterium and Bacteroides.
At the phylum level, we observed that cows that developed metritis had higher relative abundance of Bacteroidetes and lower relative abundances of Firmicutes, Tenericutes, and "others" at 7 DIM and higher relative abundance of Tenericutes, Actinobacteria, and "others" at 35 DIM. Furthermore, the Chao1 and Shannon indices were decreased in metritic cows at 7 DIM. These changes in metritic cows agree with the previous literature evaluating the uterine microbiome (Jeon et al., 2015; Knudsen et al., 2016; Bicalho et al., 2017b; Sicsic et al., 2018) or the vaginal microbiome (Bicalho et al., 2017a). The uterine microbiome has been shown to change rapidly from 0 to 6 DIM, mainly due to a decreasing abundance of Proteobacteria and increasing abundance of Bacteroidetes and Fusobacteria, which were dominant in metritic cows (Jeon et al., 2015; Knudsen et al., 2016; Bicalho et al., 2017b; Sicsic et al., 2018). Moreover, Fusobacterium, Bacteroides, and Porphyromonas work synergistically with other bacteria such as Helcococcus, Filifactor, and Prevotella to cause metritis in dairy cows (Jeon et al., 2015). To our knowledge, this is the first time that cows that developed metritis were shown to have a greater relative abundance of Fusobacterium at calving. We recently also showed that the absolute abundance of uterine total bacteria, Fusobacterium necrophorum, Bacteroides pyogenes, Porphyromonas levii, Helcococcus ovis, and Prevotella melaninogenica were increased at 6 DIM in cows with metritis compared with cows without metritis (Cunha et al., 2018). Interestingly, 2 common pathogens described as being involved in the pathogenesis of uterine diseases; namely, Escherichia coli and Trueperella pyo-
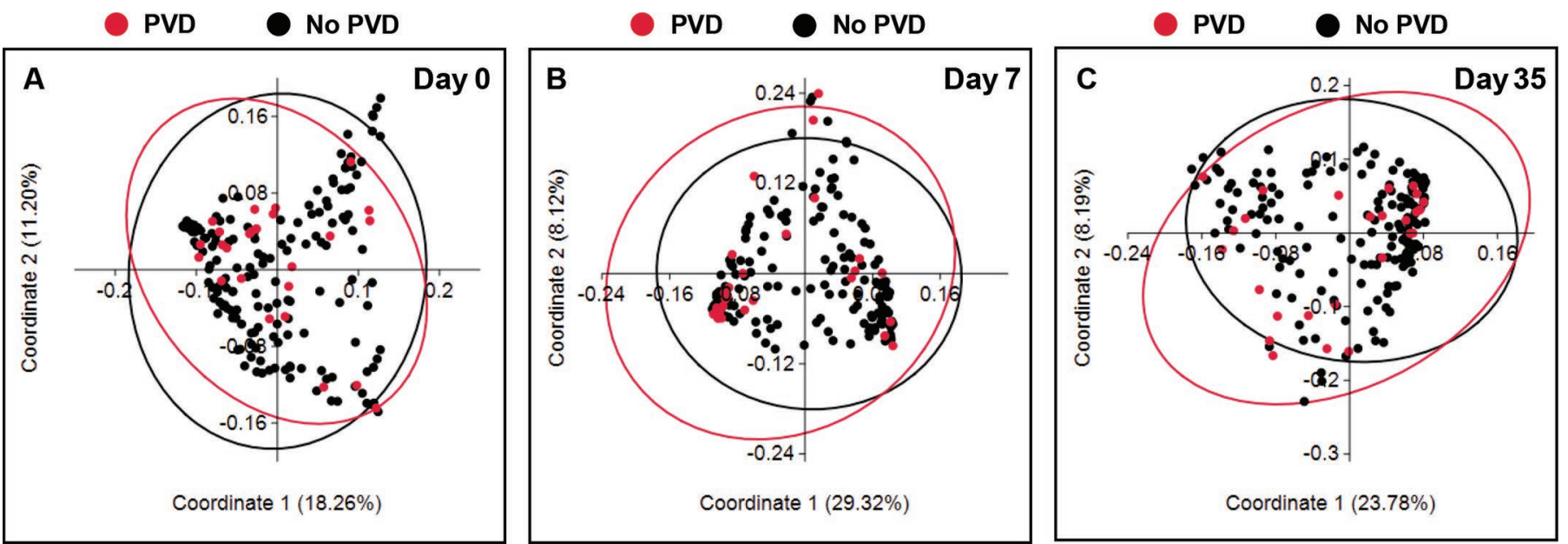

Figure 6. Evaluation of the association between the reproductive tract microbiome at 0 (A), 7 (B) and 35 (C) DIM and the diagnosis of purulent vaginal discharge (PVD) at 35 DIM using principal coordinate analysis of Bray-Curtis dissimilarity using the lowest taxonomic classification available. There was no association between PVD diagnosis at 35 DIM and the reproductive tract microbiome at 0 DIM $(P=0.28)$, but there was an association between PVD diagnosis at 35 DIM and the reproductive tract microbiome at $7(P=0.03)$ and 35 DIM $(P=0.02)$. 


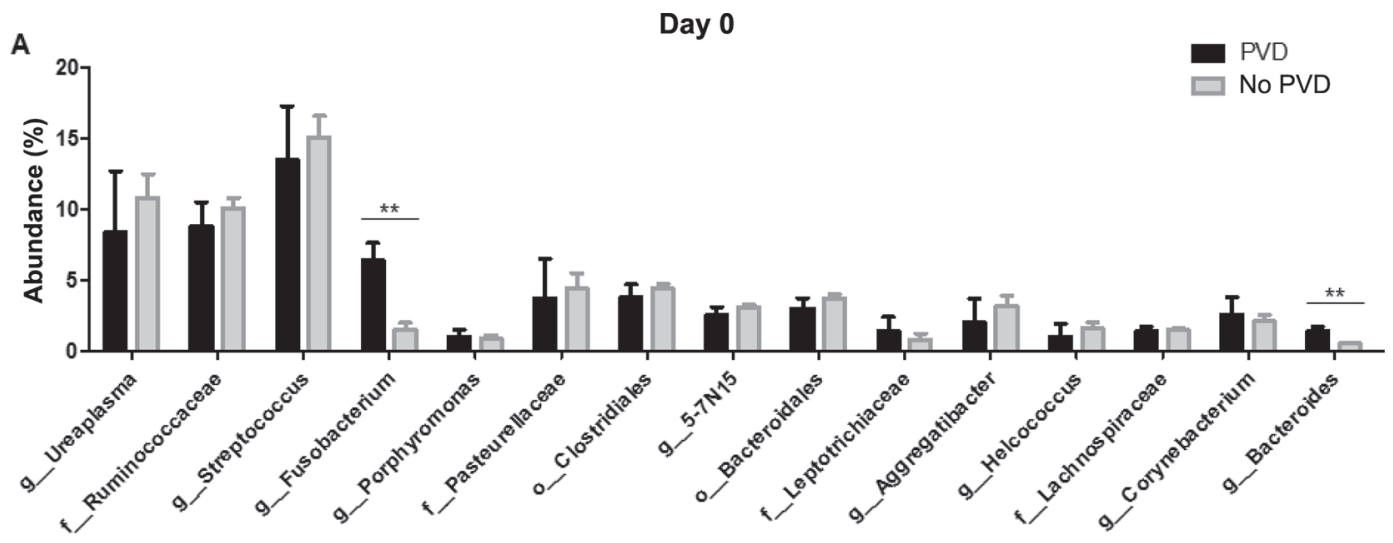

Day 7
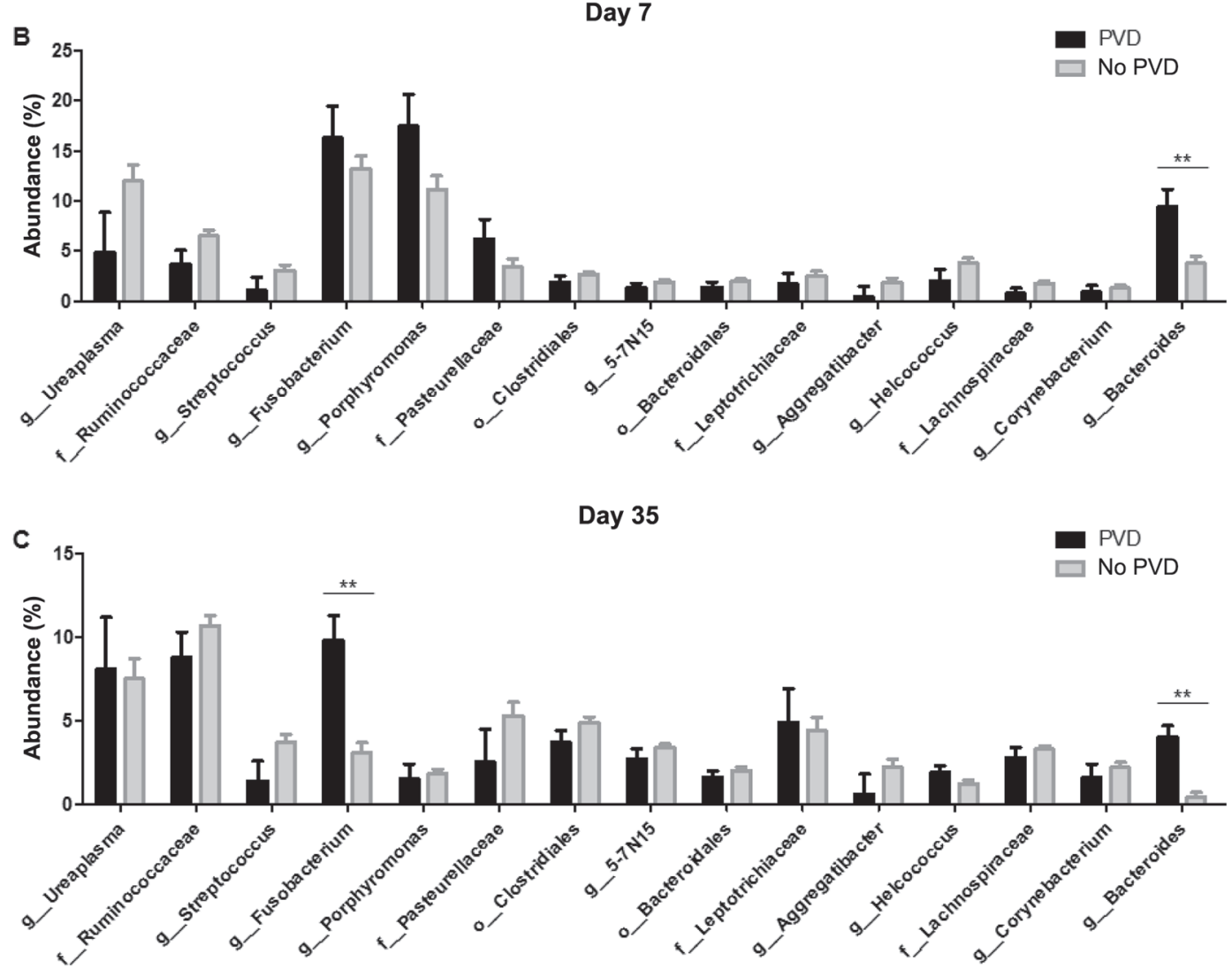

Figure 7. Mean relative abundance of the 15 most prevalent bacterial taxa (letters preceding name indicate taxon: $\mathrm{g}=$ genus, $\mathrm{f}=$ family, $\mathrm{o}$ $=$ order) found in the reproductive tract at 0 (A), 7 (B) and 35 (C) DIM in cows diagnosed or not with purulent vaginal discharge (PVD) at 35 DIM. **Significant $(P \leq 0.01)$ difference between groups. Error bars represent SEM.

genes (Williams et al., 2005; Sheldon et al., 2009), were found to have low overall absolute abundance that was similar between metritic and healthy cows (Cunha et al., 2018). This indicates that E. coli and T. pyogenes are not a significant component of the mixed infection of the uterus at 6 DIM, which is in line with our findings herein and with other recent $16 \mathrm{~S}$ metagenomic studies (Jeon et al., 2015; Knudsen et al., 2016; Bicalho et al., 2017b; Sicsic et al., 2018). It is possible that $E$. coli with specific virulence factors such as fimH (Bicalho et al., 2010; Sheldon et al., 2010) play a more significant role in the development of metritis in the first few days after calving, when the uterine environment is less conducive to obligate anaerobe proliferation. As a facultative anaerobic organism, E. coli may colonize the uterine lumen early after calving, although at low abundance, and act as a pioneer organism until the changes in the uterine environment create a competi- 
tive disadvantage that allows other anaerobes to thrive. Conversely, although the microbiome of endometritic cows is dominated by Bacteroidetes and Fusobacteria, T. pyogenes may be more important in the development of endometritis (Machado et al., 2012). Herein, we saw that metritic cows had a significant increase in the relative abundance of Actinobacteria, which includes $T$. pyogenes, at 35 DIM, whereas endometritic cows had a numeric increase compared with cows that did not develop uterine disease.

Decreased richness and diversity of uterine microbiota in metritic cows was also observed in our previous work (Jeon et al., 2015, 2016). Recent work evaluating the microbiome of the vagina observed a similar pattern of change in the microbiome of metritic cows as observed in uterine samples, such as a greater relative abundance of total bacteria, Fusobacteria, and Bacteroidetes in metritic cows compared with cows without metritis at 7 DIM (Bicalho et al., 2017a). The similarity between uterine and vaginal samples is likely because most of the discharge found in the vagina postpartum is likely derived from the uterus. Lower relative abundances of Ureaplasma and Ruminococcaceae at 7 DIM in metritic cows also agree with our previous observation that $R u$ minococcus and Ureaplasma were associated with uterine health (Jeon et al., 2015). Lower relative abundance of Clostridiales in metritic cows is a novel finding; however, we had observed that Clostridium was negatively correlated with Bacteroides, indicating, therefore, that it was not part of the dysbiosis observed in metritic cows. For the most part, the changes observed in cows that developed PVD were similar to those observed in cows that developed metritis, likely because of the high correlation between metritis and PVD.

Interestingly, antibiotic treatment of cows with metritis was not associated with their vaginal microbiome structure at 7 or 35 DIM. This finding agrees with a recent report in which we showed that the uterine microbiome structure was not markedly changed $6 \mathrm{~d}$ after treatment with antibiotics, although there were some minor differences depending on the antibiotic used (Jeon et al., 2018). Hence, it is likely that antibiotics affect the absolute number of microbes in the reproductive tract rather than their relative abundance.

\section{CONCLUSIONS}

In summary, PEG treatment had no effect on the microbiome found in the vagina but the vaginal microbiome was associated with the development of uterine disease. In general, cows that developed metritis and PVD had reduced richness and diversity of the vaginal microbiome and an increased relative abundance of the phylum Bacteroidetes and the genera Fusobacterium, Porphyromonas, and Bacteroides.

\section{ACKNOWLEDGMENTS}

This study was funded by Elanco Animal Health (Greenfield, IN).

\section{REFERENCES}

Bicalho, M. L. S., V. S. Machado, C. H. Higgins, F. S. Lima, and R. C. Bicalho. 2017b. Genetic and functional analysis of the bovine uterine microbiota. Part I: Metritis versus healthy cows. J. Dairy Sci. 100:3850-3862.

Bicalho, M. L. S., T. Santin, M. X. Rodrigues, C. E. Marques, S. F. Lima, and R. C. Bicalho. 2017a. Dynamics of the microbiota found in the vaginas of dairy cows during the transition period: Associations with uterine diseases and reproductive outcome. J. Dairy Sci. 100:3043-3058.

Bicalho, R. C., V. S. Machado, M. L. Bicalho, R. O. Gilbert, A. G. Teixeira, L. S. Caixeta, and R. V. Pereira. 2010. Molecular and epidemiological characterization of bovine intrauterine Escherichia coli. J. Dairy Sci. 93:5818-5830.

Bokulich, N. A., S. Subramanian, J. J. Faith, D. Gevers, J. I. Gordon, R. Knight, D. A. Mills, and J. G. Caporaso. 2013. Quality-filtering vastly improves diversity estimates from Illumina amplicon sequencing. Nat. Methods 10:57-59.

Caporaso, J. G., J. Kuczynski, J. Stombaugh, K. Bittinger, F. D Bushman, E. K. Costello, N. Fierer, A. G. Pena, J. K. Goodrich, J. I. Gordon, G. A. Huttley, S. T. Kelley, D. Knights, J. E. Koenig, R. E. Ley, C. A. Lozupone, D. McDonald, B. D. Muegge, M. Pirrung, J. Reeder, J. R. Sevinsky, P. J. Turnbaugh, W. A. Walters, J. Widmann, T. Yatsunenko, J. Zaneveld, and R. Knight. 2010. QIIME allows analysis of high-throughput community sequencing data. Nat. Methods 7:335-336.

Caporaso, J. G., C. L. Lauber, W. A. Walters, D. Berg-Lyons, J. Huntley, N. Fierer, S. M. Owens, J. Betley, L. Fraser, M. Bauer, N. Gormley, J. A. Gilbert, G. Smith, and R. Knight. 2012. Ultrahigh-throughput microbial community analysis on the Illumina HiSeq and MiSeq platforms. ISME J. 6:1621-1624.

Capper, J. L., R. A. Cady, and D. E. Bauman. 2009. The environmental impact of dairy production: 1944 compared with 2007. J. Anim. Sci. 87:2160-2167.

Cha, E., D. Bar, J. A. Hertl, L. W. Tauer, G. Bennett, R. N. González, Y. H. Schukken, F. L. Welcome, and Y. T. Gröhn. 2011. The cost and management of different types of clinical mastitis in dairy cows estimated by dynamic programming. J. Dairy Sci. 94:4476-4487.

Chakravorty, S., D. Helb, M. Burday, N. Connell, and D. Alland. 2007. A detailed analysis of $16 \mathrm{~S}$ ribosomal RNA gene segments for the diagnosis of pathogenic bacteria. J. Microbiol. Methods 69:330-339.

Cunha, F., S. J. Jeon, R. Daetz, A. Vieira-Neto, J. Laporta, K. C. Jeong, A. F. Barbet, C. A. Risco, and K. N. Galvão. 2018. Quantifying known and emerging uterine pathogens, and evaluating their association with metritis and fever in dairy cows. Theriogenology $114: 25-33$

Edgar, R. C. 2010. Search and clustering orders of magnitude faster than BLAST. Bioinformatics 26:2460-2461.

Galvão, K. N., and J. E. P. Santos. 2014. Recent advances in the immunology and uterine microbiology of healthy cows and cows that develop uterine disease. Turk. J. Vet. Anim. Sci. 38:577-588.

Gilbert, J. A., F. Meyer, D. Antonopoulos, P. Balaji, C. T. Brown, N. Desai, J. A. Eisen, D. Evers, D. Field, W. Feng, D. Huson, J. Jansson, R. Knight, J. Knight, E. Kolker, K. Konstantindis, J. Kostka, N. Kyrpides, R. Mackelprang, A. McHardy, C. Quince, J. Raes, A. Sczyrba, A. Shade, and R. Stevens. 2010. Meeting report: The terabase metagenomics workshop and the vision of an Earth microbiome project. Stand. Genomic Sci. 3:243-248. 
Goff, J. P., and R. L. Horst. 1997. Physiological changes at parturition and their relationship to metabolic disorders. J. Dairy Sci. $80: 1260-1268$.

Goshen, T., and N. Y. Shpigel. 2006. Evaluation of intrauterine antibiotic treatment of clinical metritis and retained fetal membranes in dairy cows. Theriogenology 66:2210-2218.

Groopman, J. E., J. M. Molina, and D. T. Scadden. 1989. Hematopoietic growth factors. Biology and clinical applications. N. Engl. J. Med. 321:1449-1459.

Hammon, D. S., I. M. Evjen, T. R. Dhiman, J. P. Goff, and J. L. Walters. 2006. Neutrophil function and energy status in Holstein cows with uterine health disorders. Vet. Immunol. Immunopathol. 113:21-29.

Hassfurther, R. L., T. N. TerHune, and P. C. Canning. 2015. Efficacy of polyethylene glycol-conjugated bovine granulocyte colonystimulating factor for reducing the incidence of naturally occurring clinical mastitis in periparturient dairy cows and heifers. Am. J. Vet. Res. 76:231-238.

Hussain, A. M. 1989. Bovine uterine defense mechanism: A review. Zentralbl. Veterinarmed. B 36:641-651.

Jeon, S. J., F. Cunha, X. Ma, N. Martinez, A. Vieira-Neto, R. Daetz, R. C. Bicalho, S. Lima, J. E. Santos, K. C. Jeong, and K. N. Galvão. 2016. Uterine microbiota and immune parameters associated with fever in dairy cows with metritis. PLoS One 11:e0165740.

Jeon, S. J., F. S. Lima, A. Vieira-Neto, V. S. Machado, S. F. Lima R. C. Bicalho, J. E. P. Santos, and K. N. Galvão. 2018. Shift of uterine microbiota associated with antibiotic treatment and cure of metritis in dairy cows. Vet. Microbiol. 214:132-139.

Jeon, S. J., A. Vieira-Neto, M. Gobikrushanth, R. Daetz, R. D. Mingoti, A. C. Parize, S. L. de Freitas, A. N. da Costa, R. C. Bicalho, S. Lima, K. C. Jeong, and K. N. Galvão. 2015. Uterine microbiota progression from calving until establishment of metritis in dairy cows. Appl. Environ. Microbiol. 81:6324-6332.

Kimura, K., J. P. Goff, P. Canning, C. Wang, and J. A. Roth. 2014. Effect of recombinant bovine granulocyte colony-stimulating factor covalently bound to polyethylene glycol injection on neutrophil number and function in periparturient dairy cows. J. Dairy Sci. 97:4842-4851.

Knudsen, L. R., C. C. Karstrup, H. G. Pedersen, Ø. Angen, J. S Agerholm, E. L. Rasmussen, T. K. Jensen, and K. Klitgaard. 2016. An investigation of the microbiota in uterine flush samples and endometrial biopsies from dairy cows during the first 7 weeks postpartum. Theriogenology 86:642-650.

LeBlanc, S. J., T. F. Duffield, K. E. Leslie, K. G. Bateman, G. P. Keefe, J. S. Walton, and W. H. Johnson. 2002. Defining and diagnosing postpartum clinical endometritis and its impact on reproductive performance in dairy cows. J. Dairy Sci. 85:2223-2236.

Machado, V. S., G. Oikonomou, M. L. Bicalho, W. A. Knauer, R. Gilbert, and R. C. Bicalho. 2012. Investigation of postpartum dairy cows' uterine microbial diversity using metagenomic pyrosequencing of the 16S rRNA gene. Vet. Microbiol. 159:460-469.

McDonald, D., M. N. Price, J. Goodrich, E. P. Nawrocki, T. Z. DeSantis, A. Probst, G. L. Andersen, R. Knight, and P. Hugenholtz. 2012. An improved Greengenes taxonomy with explicit ranks for ecological and evolutionary analyses of bacteria and archaea. ISME J. 6:610-618.

NRC. 2001. Nutrient Requirements of Dairy Cattle. 7th rev. ed. Natl. Acad. Press, Washington, DC.
Nickerson, S. C., W. E. Owens, and J. L. Watts. 1989. Effects of recombinant granulocyte colony-stimulating factor on Staphylococcus aureus mastitis in lactating dairy cows. J. Dairy Sci. 72:3286-3294.

Nicola, N. A., D. Metcalf, M. Matsumoto, and G. R. Johnson. 1983. Purification of a factor inducing differentiation in murine myelomonocytic leukemia cells. Identification as granulocyte colonystimulating factor. J. Biol. Chem. 258:9017-9023.

Overton, M., and J. Fetrow. 2008. Economics of postpartum uterine health. Pages 39-43 in Proc. Dairy Cattle Reproduction Council (DCRC) Convention, Omaha, NE. DCRC, Hartland, WI.

Paape, M. J., D. D. Bannerman, X. Zhao, and J. W. Lee. 2003. The bovine neutrophil: Structure and function in blood and milk. Vet. Res. 34:597-627.

Ruiz, R., L. O. Tedeschi, and A. Sepulveda. 2017. Investigation of the effect of pegbovigrastim on some periparturient immune disorders and performance in Mexican dairy herds. J. Dairy Sci. 100:33053317.

Santos, J. E., R. S. Bisinotto, E. S. Ribeiro, F. S. Lima, L. F. Greco, C. R. Staples, and W. W. Thatcher. 2010. Applying nutrition and physiology to improve reproduction in dairy cattle. Soc. Reprod. Fertil. Suppl. 67:387-403.

Sheldon, I. M., J. Cronin, L. Goetze, G. Donofrio, and H. J. Schuberth. 2009. Defining postpartum uterine disease and the mechanisms of infection and immunity in the female reproductive tract in cattle. Biol. Reprod. 81:1025-1032.

Sheldon, I. M., A. N. Rycroft, B. Dogan, M. Craven, J. J. Bromfield, A. Chandler, M. H. Roberts, S. B. Price, R. O. Gilbert, and K. W. Simpson. 2010. Specific strains of Escherichia coli are pathogenic for the endometrium of cattle and cause pelvic inflammatory disease in cattle and mice. PLoS One 5:e9192.

Sicsic, R., T. Goshen, R. Dutta, N. Kedem-Vaanunu, V. KaplanShabtai, Z. Pasternak, Y. Gottlieb, N. Y. Shpigel, and T. Raz. 2018. Microbial communities and inflammatory response in the endometrium differ between normal and metritic dairy cows at 5-10 days post-partum. Vet. Res. 49:77.

Souza, L. M., T. C. Boone, J. Gabrilove, P. H. Lai, K. M. Zsebo, D. C. Murdock, V. R. Chazin, J. Bruszewski, H. Lu, and K. K. Chen. 1986. Recombinant human granulocyte colony-stimulating factor: effects on normal and leukemic myeloid cells. Science 232:61-65.

USDA-NASS. 2017. Dairy and Poultry Statistics. Accessed Feb. 5 , 2019. https://www.nass.usda.gov/Publications/Ag_Statistics/ 2017/Chapter08.pdf.

Williams, E. J., D. P. Fischer, D. U. Pfeiffer, G. C. England, D. E. Noakes, H. Dobson, and I. M. Sheldon. 2005. Clinical evaluation of postpartum vaginal mucus reflects uterine bacterial infection and the immune response in cattle. Theriogenology 63:102-117.

Zinicola, M., H. Korzec, A. G. V. Teixeira, E. K. Ganda, L. Bringhenti, A. C. C. H. Tomazi, R. O. Gilbert, and R. C. Bicalho. 2018. Effects of pegbovigrastim administration on periparturient diseases, milk production, and reproductive performance of Holstein cows. J. Dairy Sci. 101:11199-11217. https://doi.org/10.3168/jds 2018-14869.

Zsebo, K. M., A. M. Cohen, D. C. Murdock, T. C. Boone, H. Inoue, V. R. Chazin, D. Hines, and L. M. Souza. 1986. Recombinant human granulocyte colony stimulating factor: Molecular and biological characterization. Immunobiology 172:175-184. 